\title{
Utilization of a Duplex HybProbe Real-Time PCR to Detect and Estimate IL-28B Polymorphisms Prevalence among HIV/HCV Co-infected Patients in Hong Kong
}

Sabrina Wai-Chi To', Gilman Kit-Hang Siu², Ka-Hing Wong³, Kenny Chi-Wai Chan ${ }^{3}$, King-Tai Yuen', Hon-Man Ng' and Wing-Cheong Yam ${ }^{1 *}$

${ }^{1}$ Department of Microbiology, Queen Mary Hospital, The University of Hong Kong, Hong Kong, Special Administrative Region, China

${ }^{2}$ Department of Health Technology and Informatics, The Hong Kong Polytechnic University, Hong Kong, Special Administrative Region, China

${ }^{3}$ Integrated Treatment Centre, Special Preventive Programme, Centre of Health Protection, Department of Health, Hong Kong, Special Administrative Region, China

\begin{abstract}
Conventional treatment for chronic HCV infection relies on the combination of peg-interferon and ribavirin therapy. Both interleukin-28B (IL-28B) polymorphisms and HCV genotypes serve as the strongest predictive values for therapeutic prognosis. The treatment regimens for HIV/HCV co-infected patients are more complex and dependent on various host immune and viral factors. A rapid and cost-effective IL-28B genotyping tool is therefore crucial to assist clinicians on better patient management. This study aimed to evaluate the performance of a newly developed HybProbe duplex real-time PCR assay in detecting IL-28B polymorphisms on rs12979860 and rs8099917, and to estimate the prevalence of IL-28B polymorphisms among HIV/HCV co-infected patients in Hong Kong. A total of $88 \mathrm{HIV} / \mathrm{HCV}$ co-infected patients were recruited at the Integrated Treatment Centre during 2009 to 2014. IL28B polymorphisms on rs 12979860 and rs 8099917 were determined by an in-house HybProbe assay with melting curve analysis. For assay evaluation, IL-28B polymorphisms of 46 samples with diverse HIV/HCV genotypes were confirmed by Sanger sequencing. Both in-house HybProbe assay and sequencing results for IL28B polymorphisms determination were completely concordant. Among the $88 \mathrm{HIV} / \mathrm{HCV}$ co-infected, the frequency of rs 12979860 wildtype $(\mathrm{C} / \mathrm{C})$ was $88.6 \%$, heterozygous mutant $(\mathrm{C} / \mathrm{T})$ was $9.1 \%$ and remaining $2.3 \%$ homozygous mutant $(\mathrm{T} / \mathrm{T})$. The prevalence of IL-28B polymorphisms in rs8099917 was slightly differed, which had $90.9 \%$ wild-type (T/T), $6.8 \%$ heterozygous mutant $(\mathrm{G} / \mathrm{T})$ and $2.3 \%$ homozygous mutant $(\mathrm{G} / \mathrm{G})$. This novel duplex assay could allow clinicians to make early decision on treatment option for HIV/HCV co-infected patients by detecting rs 12979860 and rs8099917 polymorphisms simultaneously.
\end{abstract}

\section{Background}

In developed countries, the morbidity and mortality in Human Immunodeficiency Virus (HIV) patients is significantly induced by chronic Hepatitis C Virus (HCV) co-infection. It is estimated to have about one-third of HIV patients co-infected with HCV. These patients usually require immediate $\mathrm{HCV}$ treatment as they tolerate poorly towards antiretroviral treatment, progress more rapidly to end-stage liver disease and often lead to death $[1,2]$.

For decades, the standard therapy for HCV infection relies on the combination of pegylated interferon and ribavirin (PEG-IFN/RBV). This treatment is known to be less effective towards HIV/HCV coinfected patients, with a lower rate of Sustained Virological Response (SVR) and a higher rate of viral relapse upon treatment completion $[3,4]$. Recent studies identified two Single Nucleotide Polymorphisms (SNPs) rs12979860 and rs8099917 that located near interleukin-28B (IL-28B) and acted as important baseline predictors for PEG-IFN/RBV treatment responses. Strong association between favourable genotypes (C/C for rs12979860 and T/T for rs8099917) and high rate of SVR were observed in several genome wide association studies [5-8].

Clinical management on HIV/HCV co-infected patients is always more complicated than mono-infected patients. Several commercial assays and sequencing are the major IL-28B polymorphisms detection methods, yet they are relatively expensive and time-consuming. This study aimed to develop a rapid and cost-effective duplex HybProbe real-time assay to detect both rs12979860 and rs8099917 SNPs in a single reaction and estimate the prevalence of IL-28B, HIV-1 and HCV subtypes among co-infected patients in this locality.

\section{Objectives}

To evaluate the performance of a newly developed HybProbe duplex real-time PCR assay in detecting IL-28B polymorphisms on rs12979860 and rs8099917, and to estimate the prevalence of IL-28B polymorphisms among HIV/HCV co-infected patients in Hong Kong.

\section{Study Design}

A total of $88 \mathrm{HIV} / \mathrm{HCV}$ co-infected patients were recruited at the Hong Kong Government Integrated Treatment Centre during 2009 to 2014. Whole blood samples were collected after patient consent. The IL-28B polymorphisms on rs12979860 and rs8099917 of all samples were detected by the HybProbe assay with melting curve analysis. The duplex PCR assay was amplified by LightCycler ${ }^{\circ}$ FastStart DNA Master HybProbe (Roche Diagnostics, Germany), with two pairs of primers and hybridization probes specifically targeting rs12979860 and rs8099917 (Table 1). For assay evaluation, IL-28B polymorphisms of 46 samples were further confirmed by Sanger sequencing. HCV viral load was quantitated by Abbott $m 2000$ RealTime HCV assay (Abbott Laboratories, USA) while HIV-1 and HCV genotypes were confirmed

*Corresponding author: Dr. W.C. Yam, Department of Microbiology, Queen Mary Hospital, The University of Hong Kong, Hong Kong Special Administrative Region, China, Tel: +852-22554821; Fax: +852-28551241; E-mail: wcyam@hkucc.hku.hk

Received June 01, 2015; Accepted June 03, 2015; Published June 06, 2015

Citation: To SWC, Siu GKH, Wong KH, Chan KCW, Yuen KT, et al. (2015) Utilization of a Duplex HybProbe Real-Time PCR to Detect and Estimate IL-28B Polymorphisms Prevalence among HIV/HCV Co-infected Patients in Hong Kong. J Med Microb Diagn 4: 194. doi:10.4172/21610703.1000194

Copyright: ( 2015 To SWC, et al., This is an open-access article distributed under the terms of the Creative Commons Attribution License, which permits unrestricted use, distribution, and reproduction in any medium, provided the original author and source are credited. 
Citation: To SWC, Siu GKH, Wong KH, Chan KCW, Yuen KT, et al. (2015) Utilization of a Duplex HybProbe Real-Time PCR to Detect and Estimate IL-28B Polymorphisms Prevalence among HIV/HCV Co-infected Patients in Hong Kong. J Med Microb Diagn 4: 194. doi:10.4172/21610703.1000194

Page 2 of 4

by our in-house methods $[9,10]$. Statistical analysis was performed by Graphpad Prism version 6(Graphpad software, California, US).

\section{Results}

Basic demographic characteristics of $\mathrm{HIV} / \mathrm{HCV}$ co-infected patients in this study are presented in Table 2 . There were 82 males (93.2\%) included in this study, with an overall median age at 39 (range $21-63$ years). Nearly $65 \%$ of the study population were Chinese with approximately $33 \%$ non-Chinese Asians. The in-house HIV-1 genotyping analysis revealed that the patients were infected by subtype B (20.5\%), CRF01_AE (47.7\%) and C or BC recombinants (15.9\%). $\mathrm{HCV}$ genotypes were detected in 71 patients, including genotype 1/1a/1b (30.7\%), 3a/3b (26.1\%) and 6a/6d/6e (23.9\%). Due to low HIV-1 or HCV viral load at sample collection, about $15-20 \%$ of the co-infected patients were unable to have either or both viral genotyping results.
The HybProbe assay performance was evaluated among 46 coinfected samples, using Sanger sequencing as gold standard. Diverse HIV-1 subtypes (5 subtype B, 3 subtype C, 29 CRF01_AE, 4 CRF07_BC, 1 CRF08_BC and 4 undetermined) and HCV genotypes (2 genotype 1,9 genotype 1a, 6 genotype 1b, 2 genotype $3 a, 1$ genotype $3 b$, 9 genotype $6 a, 1$ genotype $6 \mathrm{~d}, 3$ genotype $6 \mathrm{e}$ and 13 undetermined) samples were included for evaluation. Sanger sequencing required two individual reactions and manual sequence proof-reading whereas in-house HybProbe assay required melting curve analysis to interpret the SNPs on rs12979860 and rs8099917. Rs12979860 C/C wild-type had a melting peak at $64^{\circ} \mathrm{C} \pm 1^{\circ} \mathrm{C}$ while $\mathrm{T} / \mathrm{T}$ mutant had a lower melting temperature at $54^{\circ} \mathrm{C} \pm 1^{\circ} \mathrm{C}$ (Figure 1A). For rs8099917, $\mathrm{T} / \mathrm{T}$ wild-type melted at $64^{\circ} \mathrm{C} \pm 1^{\circ} \mathrm{C}$ whereas mutant $\mathrm{G} / \mathrm{G}$ melted at $57^{\circ} \mathrm{C} \pm 1^{\circ} \mathrm{C}$ (Figure 1B). Heterozygous mutants (C/T of rs12979860 and T/G of rs8099917) could be typically interpreted when both melting peaks of wild-type and mutant were seen. Both systems

\begin{tabular}{|l|l|}
\hline \multicolumn{2}{|c|}{ rs 12979860} \\
\hline Forward Primer & 5'- GCGCTTATCGCATACGGCTA - 3' \\
\hline Reverse Primer & 5'- TCACAGAAGGGAGCCCTGCC - 3' \\
\hline Sensor Probe & 5'- CGAAGGCGCGAACCAGG-Fluorescein - 3' \\
\hline Anchor Probe & 5'- LC Red 640 - TGAATTGCTCCGCGCTCCC-Phosphate - 3' \\
\hline & rs8099917 \\
\hline Forward Primer & 5'- AAGTAACACTTGTTCCTTGTAAAAGATTCC - 3' \\
\hline Reverse Primer & 5'- CGCTATAATTAAAGATGTGGGAGAATGCAA - 3' \\
\hline Sensor Probe & 5' - CCTTTCTGTGAGCAATITCACCCAA- Fluorescein - 3' \\
\hline Anchor Probe & 5' - LC Red 705 - TGGAACCATGCTGTATACAGTTTGGTAGC-Phosphate - 3' \\
\hline
\end{tabular}

$\underline{\underline{C}}$ or I indicates SNPs position.

Table 1: Primers and probes for IL-28B Genotyping assays.

\begin{tabular}{|c|c|c|c|c|c|c|c|}
\hline & \multirow{2}{*}{$\begin{array}{l}\text { Total } \\
\mathrm{n}(\%)\end{array}$} & \multicolumn{2}{|c|}{ rs12979860 } & \multirow[t]{2}{*}{$P$-value* } & \multicolumn{2}{|c|}{ rs8099917 } & \multirow[t]{2}{*}{$P$-value* } \\
\hline & & $\mathrm{CC}$ & $\mathrm{CT} / \mathrm{TT}$ & & TT & TG/GG & \\
\hline & & $(n=78)$ & $(n=10)$ & & $(n=80)$ & $(n=8)$ & \\
\hline Gender & & & & 0.5260 & & & 0.4455 \\
\hline Male & $82(93.2)$ & $73(83.0)$ & $9(10.2)$ & & $75(85.2)$ & $7(8.0)$ & \\
\hline Female & $6(6.8)$ & $5(5.7)$ & $1(1.1)$ & & $5(5.7)$ & $1(1.1)$ & \\
\hline Ethnicity & & & & 0.7117 & & & 0.2572 \\
\hline Chinese & $57(64.8)$ & $50(56.8)$ & $7(8.0)$ & & $50(56.8)$ & $7(8.0)$ & \\
\hline Asian & $29(33.0)$ & $27(30.7)$ & $2(2.3)$ & & $28(31.8)$ & $1(1.1)$ & \\
\hline Undetermined & $2(2.3)$ & $1(1.1)$ & $1(1.1)$ & & $1(1.1)$ & $1(1.1)$ & \\
\hline Risk of Infection & & & & N/A & & & N/A \\
\hline IDU & $18(20.5)$ & $17(19.3)$ & $1(1.1)$ & & $17(19.3)$ & $1(1.1)$ & \\
\hline Homosexual & $7(8.0)$ & $7(8.0)$ & -- & & $7(8.0)$ & -- & \\
\hline Heterosexual & $2(2.3)$ & $2(2.3)$ & -- & & $2(2.3)$ & -- & \\
\hline Undetermined & $61(69.3)$ & $52(59.0)$ & $9(10.2)$ & & $54(61.4)$ & $7(8.0)$ & \\
\hline HIV-1 subtypes & & & & 0.0662 & & & 0.1006 \\
\hline B & $18(20.5)$ & $13(14.8)$ & $5(5.7)$ & & $14(15.9)$ & $4(4.5)$ & \\
\hline CRF01_AE & $42(47.7)$ & $39(44.3)$ & $3(3.4)$ & & $40(45.5)$ & $2(2.3)$ & \\
\hline $\begin{array}{l}\text { C or CRF07_BC or } \\
\text { CRF08_BC }\end{array}$ & $14(15.9)$ & $13(14.8)$ & $1(1.1)$ & & $13(14.8)$ & $1(1.1)$ & \\
\hline PCR -ve & $14(15.9)$ & $13(14.8)$ & $1(1.1)$ & & $13(14.8)$ & $1(1.1)$ & \\
\hline HCV genotypes & & & & 0.1267 & & & N/A \\
\hline $1 / 1 \mathrm{a} / 1 \mathrm{~b}$ & $27(30.7)$ & $26(29.5)$ & $1(1.1)$ & & $27(30.7)$ & -- & \\
\hline $3 a / 3 b$ & $23(26.1)$ & $18(20.5)$ & $5(5.7)$ & & $18(20.5)$ & $3(3.4)$ & \\
\hline $6 a / 6 d / 6 e$ & $21(23.9)$ & $19(21.6)$ & $2(2.3)$ & & $20(22.7)$ & $1(1.1)$ & \\
\hline PCR -ve & $17(19.3)$ & $15(17.0)$ & $2(2.3)$ & & $15(17.0)$ & $2(1.1)$ & \\
\hline
\end{tabular}

N/A: Not available; -ve: negative; IDU: Intravenous drug users

*Statistics: Fisher's exact tests

Table 2: Basic demographic characteristics of HIV/HCV co-infected patients. 
Citation: To SWC, Siu GKH, Wong KH, Chan KCW, Yuen KT, et al. (2015) Utilization of a Duplex HybProbe Real-Time PCR to Detect and Estimate IL-28B Polymorphisms Prevalence among HIV/HCV Co-infected Patients in Hong Kong. J Med Microb Diagn 4: 194. doi:10.4172/21610703.1000194

1A: rs12979860 homozygous wild-type (C/C) and mutant (T/T) detection

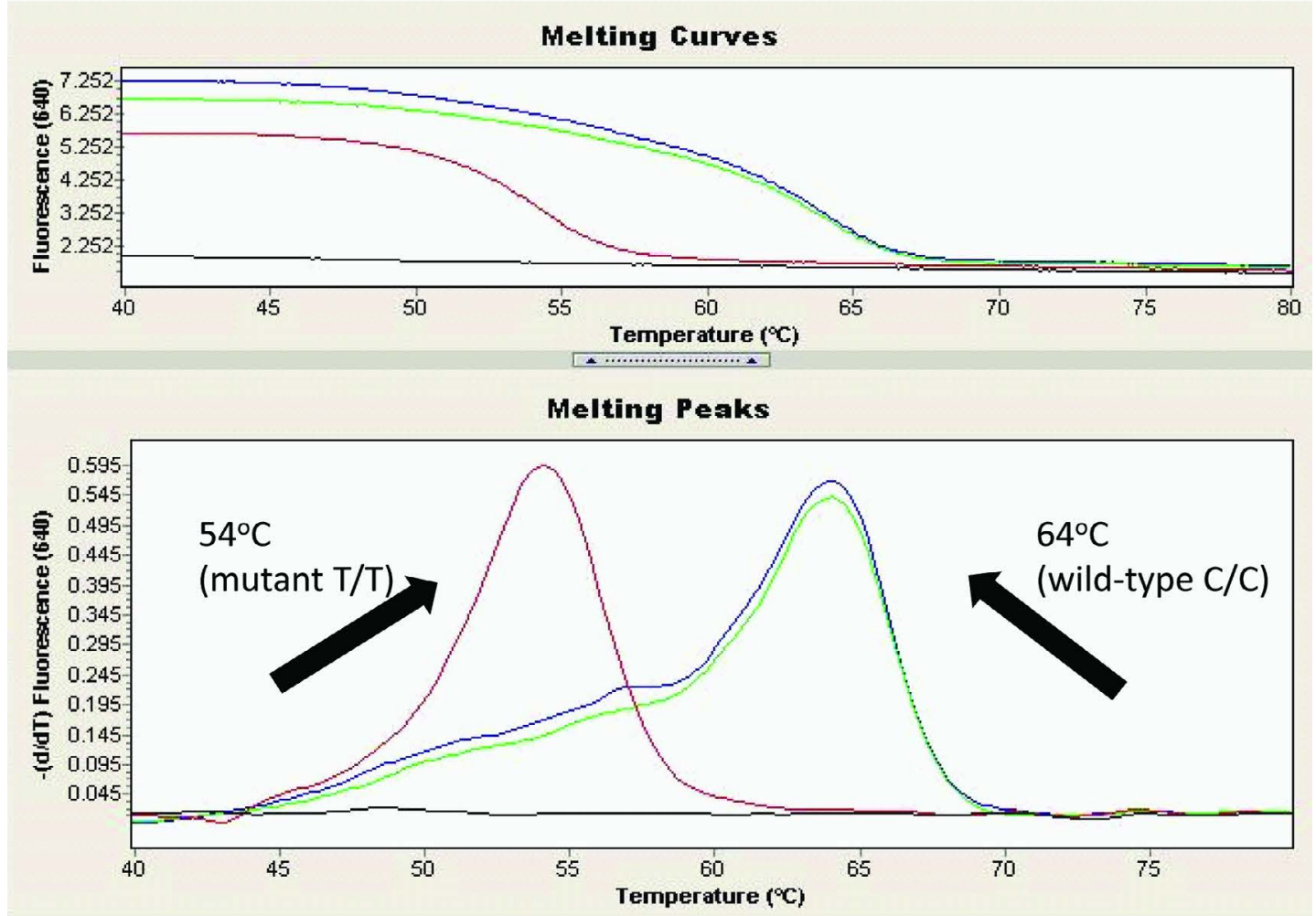

1B: rs8099917 homozygous wild-type (T/T) and mutant $(\mathrm{G} / \mathrm{G})$ detection

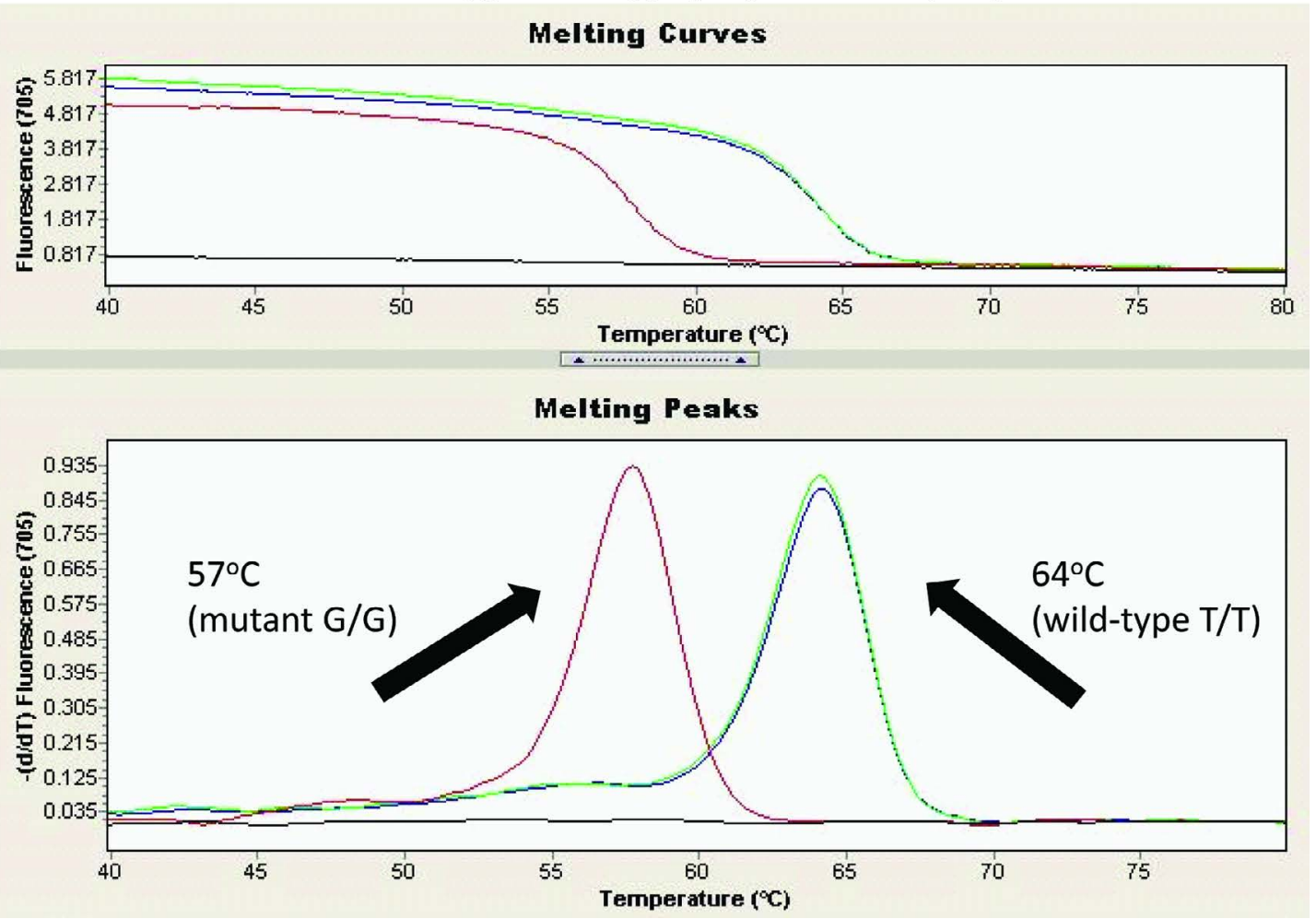

Figure 1: Melting curves and melting peaks analysis of rs12979860 (Figure 1A) and rs8099917 (Figure 1B) single nucleotide polymorphisms detection by in-house HybProbe assay. Heterozygous mutants could be interpreted when both melting peaks or wild-type and mutant were seen. 
Citation: To SWC, Siu GKH, Wong KH, Chan KCW, Yuen KT, et al. (2015) Utilization of a Duplex HybProbe Real-Time PCR to Detect and Estimate IL-28B Polymorphisms Prevalence among HIV/HCV Co-infected Patients in Hong Kong. J Med Microb Diagn 4: 194. doi:10.4172/21610703.1000194

concordantly detected two C/T and one T/T mutants of rs12979860, as well as one $\mathrm{G} / \mathrm{T}$ and one $\mathrm{G} / \mathrm{G}$ mutants of rs8099917.

Among the $88 \mathrm{HIV} / \mathrm{HCV}$ co-infected patients in this locality, the frequency of rs 12979860 wild-type $(\mathrm{C} / \mathrm{C})$ was $88.6 \%$, while the frequency of $\mathrm{C} / \mathrm{T}$ and $\mathrm{T} / \mathrm{T}$ mutants was $9.1 \%$ and $2.3 \%$ respectively. The prevalence of IL-28B polymorphisms is rs 8099917 was significantly differed $(\mathrm{P}<0.0001$, Fisher's exact tests $)$, which had $90.9 \%$ wildtype $(\mathrm{T} / \mathrm{T}), 6.8 \% \mathrm{G} / \mathrm{T}$ and $2.3 \% \mathrm{G} / \mathrm{G}$ mutants. This study revealed no association between HIV-1 subtypes, HCV genotypes, ethnicities or gender between rs12979860 or rs8099917 unfavourable genotypes. Most of the patients had concordant wild-type or mutant genotypes between both rs12979860 and rs8099917. Exceptions were seen in one Asian and one patient with undetermined ethnicity as they had unfavourable $\mathrm{C} / \mathrm{T}$ on rs12979860 but favourable wild-type $\mathrm{T} / \mathrm{T}$ on rs8099917.

\section{Discussion}

The identification of both rs12979860 and rs8099917 favourable genotypes largely enhanced the rate of SVR in HIV/ HCV co-infected patients. To our knowledge, this is the first inhouse duplex IL-28B real-time HybProbe assay that successfully identifies both rs12979860 and rs8099917 SNPs simultaneously in one single tube. The HybProbe assay used LC-RED640 and LCRED705 acceptor dyes to detect both SNPs in a single run instead of individual sequencing reactions. The melting temperatures for both SNPs interpretation were also very distinct, which was $10^{\circ} \mathrm{C}$ for rs 12979860 and $6^{\circ} \mathrm{C}$ for rs 8099917 , respectively. Traditional Sanger sequencing consists of several tedious experimental steps, including end-point PCR and capillary analysis, whereas the in-house duplex assay largely shortened the turnaround time from two days to two hours with at least $50 \%$ of running cost reduction.

The in-house HybProbe assay achieved 100\% concordant SNPs interpretation with the sequencing results. Both Chinese and Asians were included in this study, together with diverse HIV-1 and HCV genotypes, suggesting that the current assay would be largely applicable to global laboratories. Both HIV-1 subtype B and CRF01_ $\mathrm{AE}$ accounted for about $80 \%$ of local infections in Hong Kong [11]. A higher prevalence of CRF01_AE (47.7\%) was noticed in our patients, whereas the prevalence for subtype B was relatively low (20.5\%). Limited information on routes of infection was available at the time of study, though about a-fifth of the study population were infected via intravenous drug use. Conversely the prevalence of HCV genotype 1,3 and 6 were roughly similar, which was inconsistent with previous local studies, suggesting that the HIV/HCV co-infection might predominantly affect intravenous drug users with distinct epidemiological and transmission pattern. $[10,12]$.

Clinical management among HIV/HCV co-infected patients usually induced more complications due to drug-drug interaction and patients' immune status. Poor SVR rate was mainly associated with HCV genotype 1 and unfavourable IL-28B SNPs yet the information on other HCV genotypes and HIV co-infection was scarce. Two of our patients had C/T mutant on rs12979860 but wild-type T/T on rs8099917. Their treatment response towards 48-week of PEG-IFN/ RBV should be analysed in greater details to assess the impact of discordant IL-28B polymorphisms. Due to data availability, the HIV/ HCV treatment history and rate of SVR were insufficient for further investigation on the relationship between IL28B polymorphisms, $\mathrm{HIV} / \mathrm{HCV}$ genotypes and treatment responses among HIV/HCV coinfected patients.

\section{Conclusion}

This study developed a simple and efficient IL28B duplex realtime HybProbe assay with excellent performance on rs12979860 and rs8099917 SNPs detection. The rapid detection allows clinicians to make early decision on treatment option and duration. Both $\mathrm{C} / \mathrm{C}$ and T/T wild-types of rs12979860 and rs8099917 were prevalent in our locality. No significant association were observed between IL28B polymorphisms and HIV/HCV genotypes.

\section{Acknowledgement}

The study was supported by the AIDS Trust Fund of the Hong Kong Special Administrative Region Government (Grant MSS-220R). The funders had no role in study design, data collection and analysis, decision to publish, or preparation of the manuscript.

The authors are grateful to the nursing team and physicians of the Integrated Treatment Centre for their dedicated care of the patients. The opinions and assertions contained herein are private views of the authors and do not necessarily reflect those of the Centre for Health Protection, Department of Health, Hong Kong

\section{References}

1. Highleyman $L$ (2011) HIV/HCV coinfection: a new era of treatment. BETA. FallWinter 23(4): 30-47.

2. Operskalski EA, Kovacs A (2011) HIV/HCV co-infection: pathogenesis, clinica complications, treatment, and new therapeutic technologies. Curr HIVIAIDS Rep. Mar 8(1): 12-22.

3. Hadigan C, Kottilil S (2011) Hepatitis C virus infection and coinfection with human immunodeficiency virus: challenges and advancements in management JAMA 306(3): 294-301.

4. Medrano J, Barreiro P, Resino S (2009) Rate and timing of hepatitis C virus relapse after a successful course of pegylated interferon plus ribavirin in HIV infected and HIV-uninfected patients. Clin Infect Dis 49(9): 1397-1401.

5. Gonzalez SA, Keeffe EB (2011) IL-28B As a Predictor of Sustained Virologic Response in Patients with Chronic Hepatitis C Virus Infection. Gastroenterol Hepatol (N Y). 7(6): 366-373.

6. Rauch A, Kutalik Z, Descombes P (2010) Genetic variation in IL28B is associated with chronic hepatitis $\mathrm{C}$ and treatment failure: a genome-wide association study. Gastroenterology. 138(4): 1338-1345, 1345 e1331-1337.

7. Balagopal A, Thomas DL, Thio CL (2010) IL28B and the control of hepatitis C virus infection. Gastroenterology 139(6): 1865-1876.

8. Tanaka Y, Nishida N, Sugiyama M (2009) Genome-wide association of IL28B with response to pegylated interferon-alpha and ribavirin therapy for chronic hepatitis C. Nat Genet 41(10): 1105-1109.

9. Chen JH, Wong KH, Chan K (2007) Evaluation of an in-house genotyping resistance test for HIV-1 drug resistance interpretation and genotyping. J Clin Virol 39(2):125-131.

10. Lam TH, Cheng RS, Lai ST (2010) Evaluation of in-house and commercial genotyping assays for molecular typing of hepatitis $\mathrm{C}$ virus in Hong Kong. $\mathrm{Br} \mathrm{J}$ Biomed Sci 67(2): 82-85

11. (2013) Latest HIVIAIDS Statistics. Department of Health, The Government of the Hong Kong Special Administrative Region

12. Zhou DX, Tang JW, Chu IM (2006) Hepatitis C virus genotype distribution among intravenous drug user and the general population in Hong Kong. J Med Virol 78(5): 574-581.

Citation: To SWC, Siu GKH, Wong KH, Chan KCW, Yuen KT, et al. (2015) Utilization of a Duplex HybProbe Real-Time PCR to Detect and Estimate IL28B Polymorphisms Prevalence among HIV/HCV Co-infected Patients in Hong Kong. J Med Microb Diagn 4: 194. doi:10.4172/21610703.1000194 